\title{
A Novel Auto-Zero Technique for a Bio-Implantable Blood Pressure Monitoring Device
}

\author{
Mohammad Maymandi-Nejad*; Member, IEEE, Manoj Sachdev**; Senior Member, IEEE \\ * Department of Electrical Engineering, Ferdowsi University of Mashhad, Iran \\ ** Dept. of Electrical and computer Eng., University of Waterloo, Waterloo, Canada \\ maymandi@um.ac.ir,msachdev@ece.uwaterloo.ca
}

\begin{abstract}
An auto-zero (AZ) technique to cancel offset and drift in a bio-implantable integrated circuit is discussed in this paper. The implemented circuit is designed for monitoring blood pressure of transgenic mice. Due to small available volume, the power consumption is the primary design issue. In order to reduce the power consumption, techniques at the system level as well as circuit level are used. The auto-zero technique used in this IC helps reduce the complexity of the circuit blocks; which consequently results to lower power consumption. The chip is fabricated in $0.18 \mu \mathrm{m}$ standard CMOS technology. The simulation and measurement results of the implemented chip will be presented.
\end{abstract}

\section{INTRODUCTION}

Rapid advancements in silicon technology in the last few decades have opened new opportunities in the field of biomedical applications. With some modifications, the knowledge and experience obtained in the design of digital and communication circuits and systems can be used for medical applications [1, 2, 3]. Even though, power consumption is an important aspect of any portable system, in the case of bio-implantable circuits the power consumption of the implantable integrated circuit (IC) is more critical. This is because in these kinds of applications the energy source can not be replaced easily. Although there are some techniques that can provide energy to the IC from outside the body, there are some applications where these techniques can not be used. In these applications only viable way to provide energy to the implantable IC is using a miniature battery. On the other hand, commercial miniature batteries have limited capacity, and hence, demand ultra low power circuits [4].

In order to reduce the power consumption of an implantable device, new techniques should be found at the system level as well as circuit level. At the system level one approach to reduce the power is to simplify the implantable part of the system and decreasing the number of its blocks. This approach is especially effective if the power hungry blocks, like analog to digital converters (ADCs) and buffers, can be removed from the implant. This has some consequences that should be taken care of. At the circuit level, low power circuit techniques should be used. Given the low supply voltage and relatively large voltage variations of miniature batteries $(1.6 \mathrm{~V}$ down to $0.9 \mathrm{~V}$ over time) [4], the design of such circuits becomes challenging and nontrivial. In this paper we describe an auto-zeroing (AZ) technique used in a system, implemented to measure the blood pressure of transgenic mice. Using this $\mathrm{AZ}$ technique helps reduce the offset and drift, and consequently simplifies the circuit and reduces the power consumption.

The paper is organized as follows. In section 2, a short description of the system is provided and design challenges are summarized. In section 3, the low power architecture used in the implant is described. The impact of the antenna on the performance of the transmitter when the RF buffer is eliminated is explained in section 4 and the $\mathrm{AZ}$ technique to take care of this issue is described in section 5. Measurement results of the implemented chip are provided in section 6. Finally, section 7 concludes the article.

\section{SYSTEM SPECIFICATIONS}

A wireless bio-implantable system to monitor blood pressure of transgenic mice is implemented in $0.18 \mu \mathrm{m}$ CMOS technology. The system has two parts: the implant and the base station (Fig. 1). In order to measure blood pressure, the implant uses a MEMS strain gauge sensor placed in the left ventricle of the heart. An integrated circuit measures the blood pressure and transmits the data to a base station (the receiver). A miniature battery supplies the energy for the implant. Using this battery the implant should be able to operate for more than two months. Therefore, the low power consumption of the IC is crucial. The implant is capable of sending 2000 Samples/S which is approximately 200 times the heart beat rate of a mouse [5]. At the base station, the transmitted signal is detected and processed to extract the blood pressure. The data will then go to a data logger or PC for recording or further processing. The base station is implemented using off-the-shelf components since there is no limitation on its power consumption, size, and weight.

In order to design the IC two separate approaches can be taken. One is using an ADC to digitize the data 
before transmission. The ADC typically requires a relatively large amount of power. The other approach is to transmit the measured data in analog domain. This eliminates the power hungry ADC. However, the signal to noise ratio (SNR) may be inferior compare to the first approach. Hence, in this regard there is trade off between power consumption and SNR.
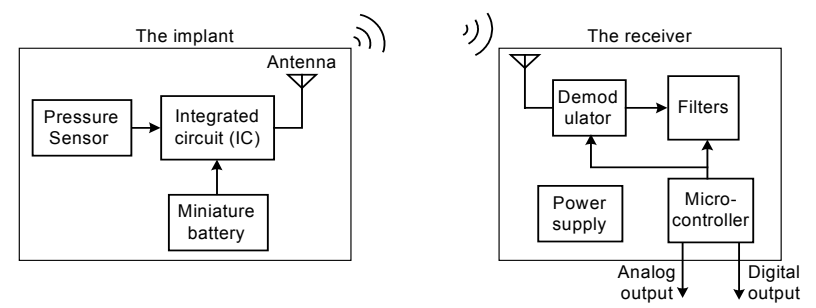

Figure 1. The schematic block diagram of the blood pressure monitoring system.

\section{LOW POWER TRANSMITTER}

Since power consumption is the primary design issue in our design we have selected analog approach to measure and transmit blood pressure data. Fig. 2 shows the block diagram of the implantable device. As can be seen in this figure, the implant is consisted of a sensor, an IC, a battery, an inductor, and an antenna. All the measurement, signal processing and transmission are done in analog domain.

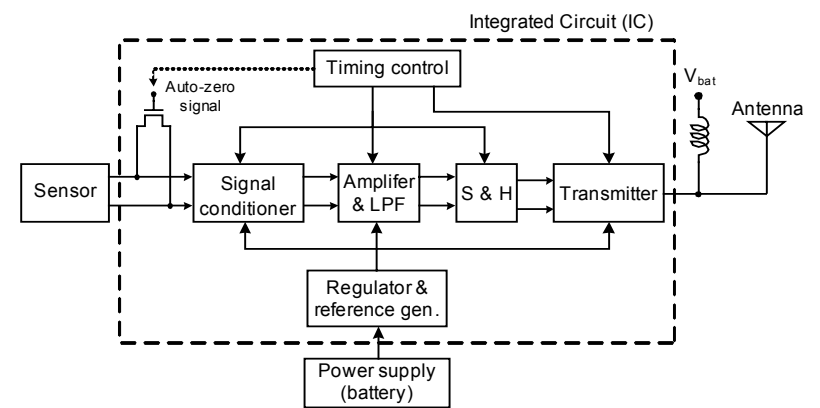

Figure 2. The schematic block diagram of the implantable device.

Also, note that a fully differential architecture is used which can help reduce the impact of noise, temperature, and other environmental variations. In order to reduce power consumption a power saving scheme is implemented in the IC. This power saving scheme works based on the fact that the implant should transmit 2000 samples per second (one sample every $500 \mu \mathrm{s}$ ). Hence, each block can be turned on for a short period of time to do its task and then be turned off to save power. The timing control block is used to control the on and off times of different blocks. Fig. 3 shows the timing diagram of the IC. We have used frequency modulation (FM) to transmit the data. In the implemented chip a VCO is used as an FM modulator. The transmitter block is consisted of a differential to single ended converter and a VCO. Typically, the output of the VCO should be amplified/buffered before being connected to the antenna. This is to isolate the antenna from the VCO so that any variation of the electrical characteristic of the antenna does not change the oscillation frequency. The $\mathrm{RF}$ amplifier or buffer is a power hungry block. In our design we have eliminated the RF buffer, and the antenna is directly connected to the output of the VCO [6]. This can reduce power consumption, however, removing the buffer has some consequences which will be explained in the next section.

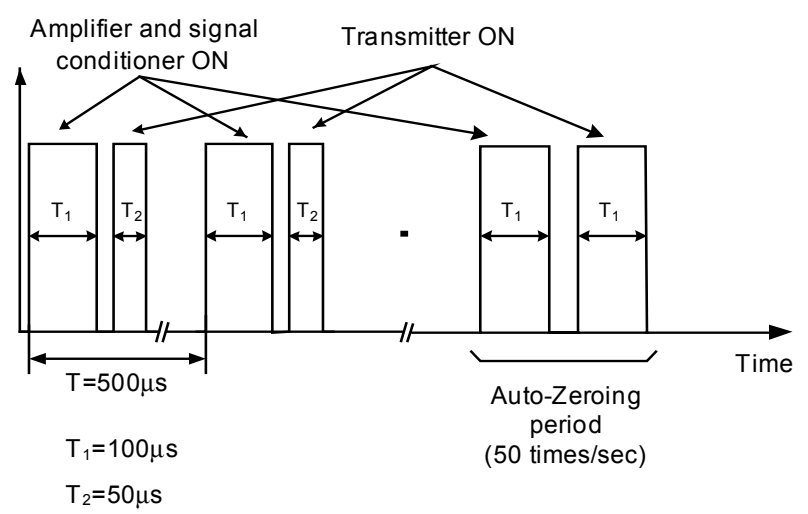

Figure 3. The timing diagram of the IC.

\section{VCO AND THE IMPACT OF THE ANTENNA}

The VCO used in our design is a Colpitts based LC oscillator as shown in Fig. 4. An off-chip inductor (L) with a high quality factor (Q) is used. Compared to a regular Colpitts oscillator, this $\mathrm{VCO}$ has an extra capacitor $\left(\mathrm{C}_{3}\right)$ and transistor $\left(\mathrm{M}_{3}\right)$. The control voltage of the VCO is applied to the gate of $\mathrm{M}_{3}$ which acts as a variable resistor. Depending upon the equivalent resistance of $\mathrm{M}_{3}$ the capacitive current passing through $\mathrm{C}_{3}$ varies which consequently changes the oscillation frequency. The range of oscillation frequency of this VCO can be obtained from Equations 1 and 2. The simulation results of the VCO is shown if Fig. 5.

$$
f_{\min }=\frac{1}{2 \pi \sqrt{L \cdot C_{1} \cdot\left(C_{2}+C_{3}\right) /\left(C_{1}+C_{2}+C_{3}\right)}}
$$

$$
f_{\max }=\frac{1}{2 \pi \sqrt{L \cdot C_{1} \cdot C_{2} /\left(C_{1}+C_{2}\right)}}
$$

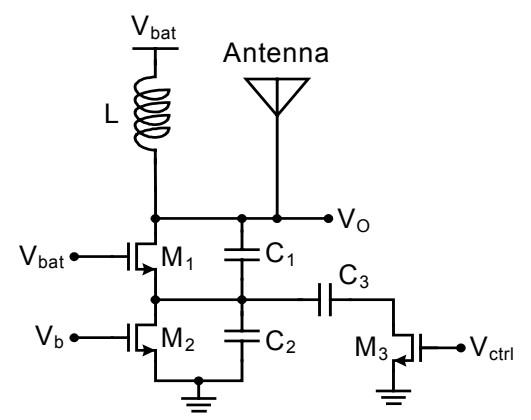

Figure 4. The schematic of the VCO.

As mentioned in the previous section, in order to reduce power consumption, we have eliminated the RF buffer between the VCO and the antenna. Elimination of this buffer causes the oscillation frequency to change as 
a result of variation in the electrical characteristic of the antenna. This effect can be considered as a capacitance in parallel to the output of the VCO. Any variation of this parasitic capacitance can change the overall LC tank capacitance, and hence the oscillation frequency.

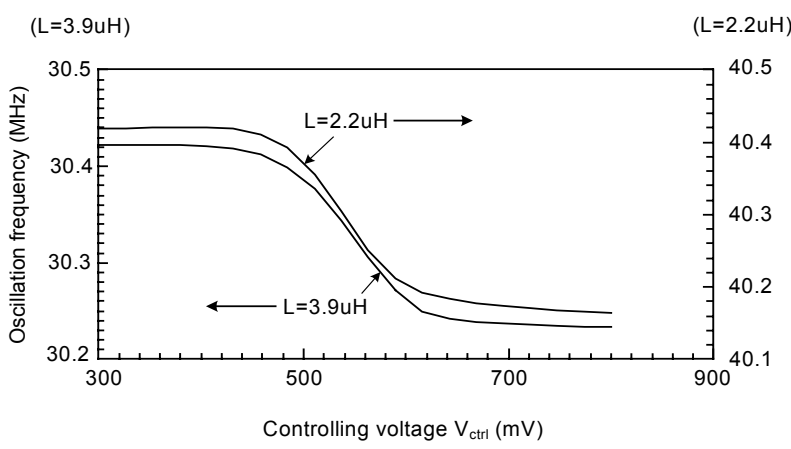

Figure 5. The oscillation frequency of the VCO versus the controlling voltage.

On the other hand, variation in the electrical characteristic of the antenna is unavoidable since the implant is to be placed in the body of a roaming mouse. An experiment to estimate the amount of frequency shift in a real environment showed that with a VCO operating at approximately $40 \mathrm{MHz}$ the frequency shift due to the impact of the antenna can be as large as $500 \mathrm{kHz}$. This amount of frequency shift should be taken care of if the RF buffer is to be emitted, otherwise the accuracy of the measurement will be greatly degraded. In order to do that, we have developed an AZ technique for the chip.

\section{THE AUTO-ZEROING TECHNIQUE}

In order to eliminate the frequency shift of the VCO due to the impact of the antenna an $\mathrm{AZ}$ technique is implemented in the designed chip. Moreover, using this technique, the offset generated in other blocks of the implantable IC as well as some of the blocks in the receiver can be reduced.

The AZ mechanism works as follows. As can be seen in the block diagram of the implant IC (Fig. 2), there is an NMOS transistor acting as a switch to short the two inputs of the signal conditioner block. This transistor is controlled by the timing control block and is turned on during AZ period. The implant transmits 2000 data samples per second. Besides the regular data, every second the implant sends 50 auto-zero data (Fig. 3). During the AZ period, the AZ transistor shorts the two output of the sensor. Under this condition the input signal is zero. However, there might be some offset in the implant's IC blocks and the VCO. This is due to the impact of the antenna, power supply variations, temperature, etc. Therefore, during this period, the signal that the base station receives is an indication of the overall offset and drift generated in the signal path. After the receiver detects the AZ signal, it adjusts its set point so that the impact of the overall offset and drift will be compensated. As can be seen in Fig. 3, the transmitter is on for a time interval of $\mathrm{T}_{2}=50 \mu \mathrm{s}$ when it sends the regular data and it is on for $\mathrm{T}_{1}=100 \mu$ s for sending AZ signal. Based on the duration of transmission, the receiver distinguishes the $\mathrm{AZ}$ signal from the regular data.

Fig. 6 shows the flow chart of the operation done in the receiver to detect the transmitted signal as well as the adjustment of the set point. After power up, the receiver goes into a detection phase when it tries to detect any near by implant that may be transmitting. This is done by sweeping the local oscillator's (LO) frequency within the specified range. Whenever, the receiver can detect the specific data pattern sent by the implant, it sets the LO's frequency. Now, the receiver is tuned to the near by implant and it starts receiving the data. In the receiver, there is a signal called Received Signal Strength Indicator (RSSI) which is an indication of the strength of the received RF signal. The duration of this pulse is measured and is used to distinguish between the $\mathrm{AZ}$ and regular data. If a regular data is received the received data is processed and is delivered to a data logger or PC. If an AZ data is received, it adjusts the set point of the receiver. This set point adjustment is done in two ways:

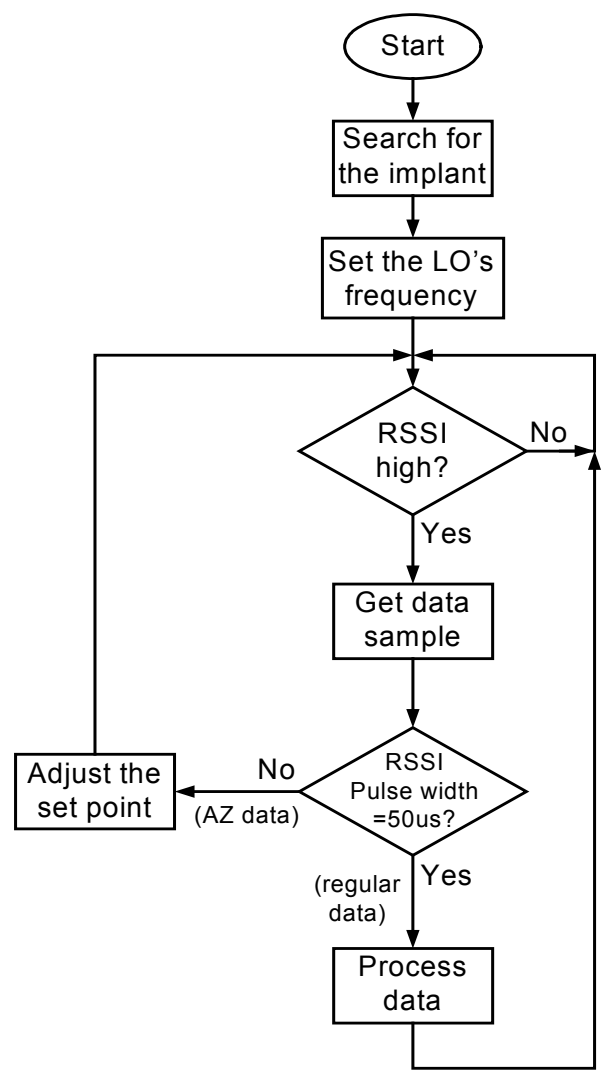

Figure 6. The flow chart of receiving data and adjusting set point.

i. The set point shift is small: Only the set point variable, which is used in the signal processing unit, is loaded with a new data.

ii. The accumulated set point shift is larger than some specific value: The LO's frequency is changed and the set point variable is adjusted accordingly (note that the modulation technique used in the implant is FM). 
Being able to change the LO's frequency is very important since the total frequency shift may be too large such that the receiver may not be tuned to the near by implant any more.

\section{MEASUREMENT RESULTS}

The implantable IC described in section 2 with the $\mathrm{AZ}$ technique described in the previous section is implemented in $0.18 \mu \mathrm{m}$ CMOS technology. Fig. 7 shows the microphotograph of the implant IC. This chip includes all the blocks shown in Fig. 2. The output of the VCO is connected to the antenna. Fig. 8 shows the output RF signal at the antenna. In Fig. 8-a the output of the VCO during two consecutive transmission periods is shown. As can be seen the transmitter is not always on to save power. Fig. 8-b shows the VCO output at the beginning of a transmission period. After the $\mathrm{VCO}$ is turned on, its output takes about $3 \mu$ s to reach the maximum amplitude. This transient response is fast enough since each transmission period is approximately $50 \mu$ s and after the VCO reaches steady state it is still has about $47 \mu$ s to transmit the data.

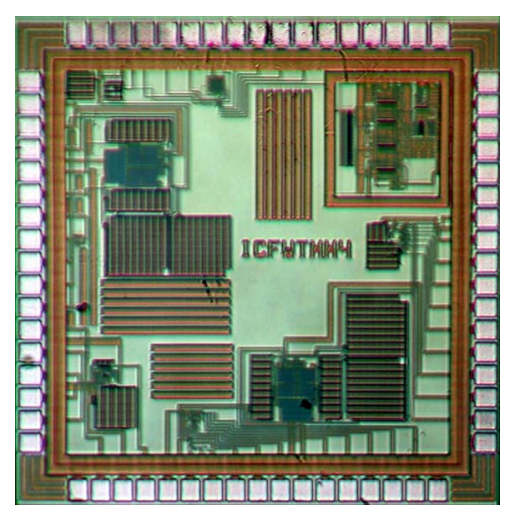

Figure 7. The implant's die microphotograph.

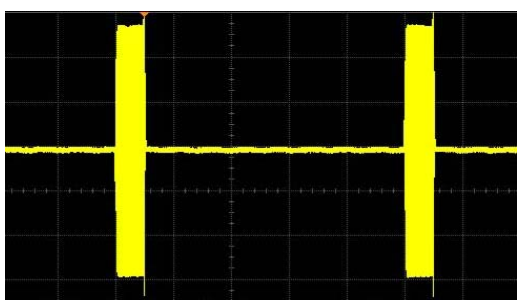

(a)

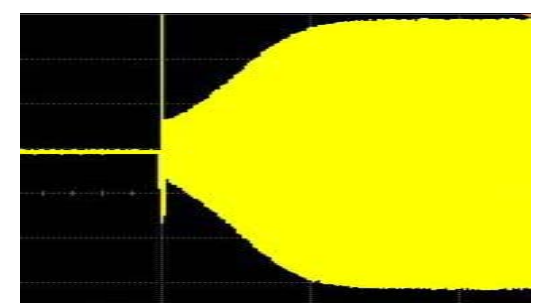

(b)

Figure 8. The output signal of the VCO during two consecutive transmissions (a), and the VCO output at the beginning of each transmission period.
In order to mimic the real conditions and to test the performance of the implant, we have used a mechanical pulser that applies pressure to the sensor of the implant similar to a mouse heart. The receiver is able to detect the transmitter, adjust its frequency, and using the AZ data to compensate the frequency shift and offset generated in the signal path. The detected signal at the receiver is shown in Fig. 9. Some of the unwanted small variations seen in this figure are due to the mechanical vibrations generated by the pulser.

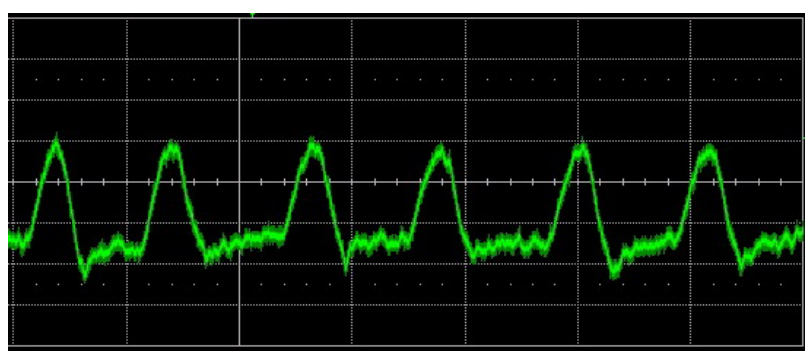

Figure 9. The pressure signal detected at the receiver.

\section{CONCLUSION}

In this paper we described a new auto-zeroing (AZ) technique which can be used for bio-implantable devices. Using this technique, it is possible to compensate any offset and drift generated in the signal path. Specifically speaking, in order to reduce power consumption of the implantable chip we have eliminated the RF buffer between the VCO and the antenna. This has the consequence of sensitizing the $\mathrm{VCO}$ to the variations of the electrical characteristics of the antenna. The proposed AZ technique can reduce the unwanted consequences of eliminating the RF buffer. An implantable chip for measuring blood pressure of mice, incorporating the $\mathrm{AZ}$ technique, is implemented in $0.18 \mu \mathrm{m}$ CMOS technology. Some of the measurement results of this chip are also reported.

\section{REFERENCES}

[1] "International Technology Roadmap for Semiconductor," Semiconductor Industry Association, 2003. Available: http/public.itrs.org.

[2] Christian Paulus, "CMOS Based Biosensors," IEEE International Workshop on Bio-Mediacal Circuits and Systems, 2004.

[3] Y. Yazawa, et al, "A Wireless Biosensing Chip for DNA Detection," IEEE International Solid State Circuits Conference, 2005.

[4] "Energizer web site," Available at: www.energizer.com.

[5] Demitrios Georgakopoulos, "Assessment of Cardiac Function in the Mouse by Pressure-Volume Relations with Applications to a Mouse Model of Familial Hypertrophic Cardiomyopathy", $J P h D$ dissertation, Johns Hopkins University, July 2000.

[6] H. Yu, K. Najafi, "Low Power Interface Circuits for Bio-Implantable Microsystems," IEEE International Solid State Circuits Conference, 2003. 